\title{
THE ROLE OF OBLIQUE-TO-SURFACE DISPOSITION OF NEIGHBOURS IN THE EMERGENCE OF SURFACE SPIN WAVES IN MAGNETIC FILMS
}

\author{
S. Mamica, R. Józefowicz and H. Puszkarski* \\ Surface Physics Division, Physics Institute, A. Mickiewicz University \\ Umultowska 85, 61-614 Poznań, Poland
}

(Received June 2, 1998)

\begin{abstract}
This work is aimed at a numerical investigation of the spin-wave energies and respective band structures throughout the two-dimensional Brillouin zone in magnetic (cubic) thin films for the surface orientations sc(001), bcc(001) and $f c c(001)$. We apply the Heisenberg localized spin model assuming exchange nearest $(\mathrm{NN})$ and next-nearest ( $\mathrm{NNN}$ ) neighbour interactions and elucidate the role of the geometrical disposition of the NN and NNN neighbourhoods for the emergence of the surface spin-waves. The necessary condition for the emergence of the surface spin-waves is turned out to require the bonds to be cut at the surface obliquely thereto irrespective of whether these "oblique" interactions are of the NN or NNN type.

PACS numbers: $75.70 .-\mathrm{i}, 75.40 . \mathrm{Gb}, 75.30 . \mathrm{Ds}$
\end{abstract}

\section{Introduction}

Recent years witnessed a revival of interest in the interaction of next-nearest neighbours (NNN) in many different fields of research. The NNN interactions are known to be responsible for interesting phase transitions (ANNNI model [1]) and have proved useful in the studies of "high- $T_{\mathrm{c}}$ cuprates" (the so-called $t-t^{\prime}-J$ model [2]). Recently, too, attention has been paid to the properties of Harper's equation with NNN coupling [3]. For a number of years, superexchange between NNN spins has been known to play an important role in thin ferromagnetic films $[4,5]$. In the paper [4] the authors studied the conditions for the emergence of surface spin-waves (SSWs) in a magnetic material with a simple cubic structure and surface cut (001); they showed that the surface fails to generate SSWs unless the range of interaction between the spins is restricted to nearest neighbours (NN)

\footnotetext{
*Corresponding autor: Professor H. Puszkarski (e-mail: henpusz@main.amu.edu.pl).
} 
only, and if the range of interaction is extended to comprise NNN, their "absence" - due to the rupture of the out-of-surface bonds - does generate SSW. However, in their conclusion the authors of Ref. [4] expressed the belief that even in the NN model surface spin-waves can exist if "the exchange interaction couples spins whose line of centres is not normal to the surface". This was a highly interesting result since in those days the emergence of SSWs was generally thought to hinge on the presence of an additional surface energy, referred to as surface anisotropy (e.g. our complementary paper [6] was published simultaneously with Ref. [4]). For the last 30 years SSWs have been studied intensely, both theoretically and experimentally, though almost exclusively in terms of surface anisotropy. The line of thought initiated by Wallis et al. has largely been neglected (for some rare exceptions, see Refs. [7-11]).

We investigated nine basic orientations of magnetic thin film surfaces, namely the structures: sc, fcc and bcc and the orientations (001), (011) and (111). In the light of our findings the conclusion drawn by Wallis et al. [4] appears to be of a universal nature; our results do indicate too that the emergence conditions of SSWs are, in fact, not sensitive to the presence of long-range interactions but rather to the geometric-structural disposition of interacting neighbours with respect to the plane of the surface cut and if the vectors connecting a spin with its neighbours are "oblique" with regard to the surface and the formation of the surface disrupts just those "oblique" interactions - then SSWs are generated irrespective of whether the interactions are $N N$ or $N N N$ type. In this paper we shall give a proof of the above for solely three (of the nine) surface orientations, namely for $\operatorname{sc}(001), b c c(001)$ and fcc(001); however, we have verified that the conclusion formulated above holds for the remaining six orientations too.

\section{The model}

We shall consider the model of a ferromagnetic thin film involving both NN and NNN exchange interactions; we assume the surface orientation to be (001) and the crystal structure as cubic. The thin film is divided (Fig. 1) into $L$ equidistant lattice planes (layers) parallel to the surface. The external magnetic field is oriented arbitrarily with regard to the film surface, and is assumed to be strong enough to stabilize the homogeneous ferromagnetic ground state (with $\gamma$ denoting the versor of the respective quantization axis). We perform our calculations within the framework of the Heisenberg localized spin model, assuming an exchange (NN and NNN) and Zeeman Hamiltonian in a standard form

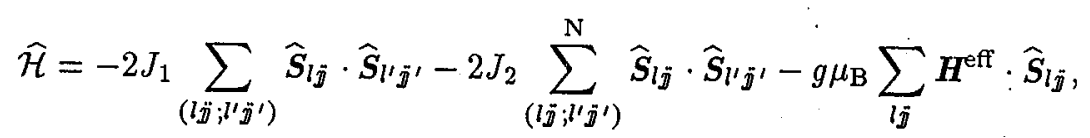

where $\boldsymbol{H}^{\text {eff }}$ comprises both external and demagnetizing fields. The summations extend over different pairs of neighbouring spins, respectively, $\sum$ stands for the summation over NN and $\sum^{N}$ over NNN. Moreover, $l j$ defines the position of a given spin, with $l$ labelling the layer (see Fig. 1) and $j$ is a two-dimensional vector defining the position of the spin in the $l$-th layer. We shall include into our considerations the following surface orientations: $\operatorname{sc}(001), \mathrm{fcc}(001)$ and $\mathrm{bcc}(001)$; 


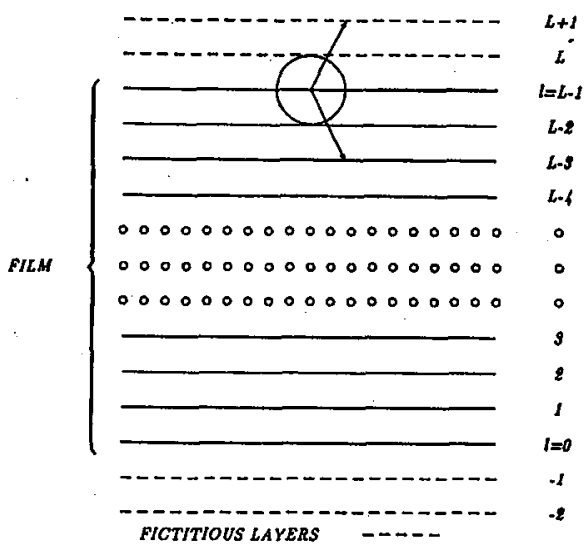

Fig. 1. Layer model of a thin ferromagnetic film.

for all of them the NNN coupling reaches at the most the second subsequent layer on each side of a given layer (this is illustrated in Fig. 1). Therefore, the NN and NNN neighbours of a given site of the layer $l$ lie in layers, which we shall label as follows: $l^{\prime}=l+n=l, l \pm 1, l \pm 2$ (thus: $n=0, \pm 1, \pm 2$ ).

We diagonalize the Hamiltonian (1) by applying the procedure described in our former paper [12]; when performing the transformation of spin operators to second quantization operators and, afterwards, the Fourier transformation in the plane of the film (this step is justified by our assumption of periodicity conditions in the directions parallel to the surface) we introduce an expression commonly referred to as a "structural sum", defined as follows:

$$
\Gamma_{n}\left( \pm k_{\|}\right)=\sum_{j^{\prime}} \exp \left[ \pm \mathrm{i} k_{\|} \cdot\left(j-j^{\prime}\right)\right]
$$

where summation over $j^{\prime}$ runs over the projections of (next/nearest) neighbours of the spin in the $l$-th plane (of the $j$-vector) belonging to the $(l+n)$-th plane (described by the $j^{\prime}$-vector). It should be noted that $\Gamma_{n}\left( \pm k_{\|}\right)$is not dependent on $j$; this is obviously a consequence of the translational invariance of the thin film in directions parallel to the film surface. In the following we discriminate structural sums over NN and NNN neighbours by denoting them as $\Gamma_{n}$ and $\Gamma_{n}^{\mathrm{N}}$, respectively. The quantity $k_{\|} \equiv\left[k_{1}, k_{2}\right]$ is a two-dimensional vector defined in the first Brillouin zone of the planar reciprocal lattice (in the film plane); utilizing the property of translational invariance parallel to the surface we express the wave-vector components $k_{1}, k_{2}$ in accordance with the usual Born-Kármán cyclic conditions. The structural sums satisfy the relations

$$
\Gamma_{-n}\left(k_{\|}\right)=\Gamma_{n}^{*}\left(k_{\|}\right)=\Gamma_{\mathrm{n}}\left(-k_{\|}\right), \quad \Gamma_{0}\left(k_{\|}\right)=\Gamma_{0}^{*}\left(k_{\|}\right) .
$$

The structural sums play an important role in determining the dynamical properties of thin film systems.

By Eq. (2), the calculation of the structural sums for a given surface cut requires the knowledge of the distribution of the projections of the neighbours of 

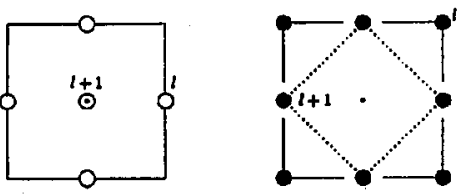

$\operatorname{sc}(001)$
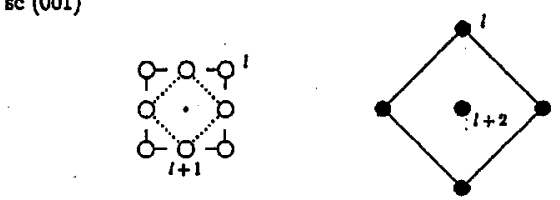

fec (001)<smiles>CC(C)(C)OC(=O)O</smiles>

$\mathrm{NN}(\mathrm{O})$

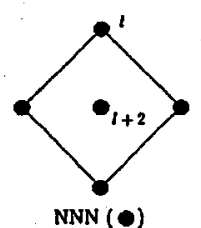

NNN (๑)

Fig. 2. The dispositions of NN and NNN neighbours for indicated three surface orientations. The neighbours of a given site (marked by a dot in the centre of each graph) are projected onto the plane $l$ in which the site lies; nearest (NN - open circles) as well as next-nearest (NNN - full circles) neighbours are taken into account. Neighbours from the plane $l$ are connected by a continuous contour, whereas neighbours from the plane $(l+1)$ are connected by a dotted contour. Neighbours from the plane $(l+2)$ are not connected by a contour since for the surface orientations under consideration those are single sites the projections of which lie in the centre of the graph. The above projections served for the calculation of the structural sums $\Gamma$ (see Table).

TABLE

Structural factors for nearest and next-nearest neighbours for three surface cuts in cubic lattices.

\begin{tabular}{c|c|c|c|c}
\hline \hline Neighbourhood & Surface & $\Gamma_{0}\left(k_{\|}\right)$ & $\Gamma_{1}\left(k_{\|}\right)$ & $\Gamma_{2}\left(k_{\|}\right)$ \\
\hline \multirow{3}{*}{ NN } & sc (001) & $2\left(\cos k_{1}+\cos k_{2}\right)$ & 1 & 0 \\
& fcc (001) & $2\left(\cos k_{1}+\cos k_{2}\right)$ & $4 \cos \frac{1}{2} k_{1} \cos \frac{1}{2} k_{2}$ & 0 \\
& bcc (001) & 0 & $4 \cos \frac{1}{2} k_{1} \cos \frac{1}{2} k_{2}$ & 0 \\
\hline \multirow{3}{*}{ NNN } & sc (001) & $4 \cos k_{1} \cos k_{2}$ & $2\left(\cos k_{1}+\cos k_{2}\right)$ & 0 \\
& fcc (001) & $4 \cos k_{1} \cos k_{2}$ & 0 & 1 \\
& bcc (001) & $2\left(\cos k_{1}+\cos k_{2}\right)$ & 0 & 1
\end{tabular}

a given site onto the plane of the surface. For the surface orientations $s c(001)$, bcc(001) and fcc(001), the NN and NNN distributions are shown in Fig. 2. We used them to calculate the sums $\Gamma_{n}$ and $\Gamma_{n}^{\mathrm{N}}$ for $n=0,1,2$ and the results are 

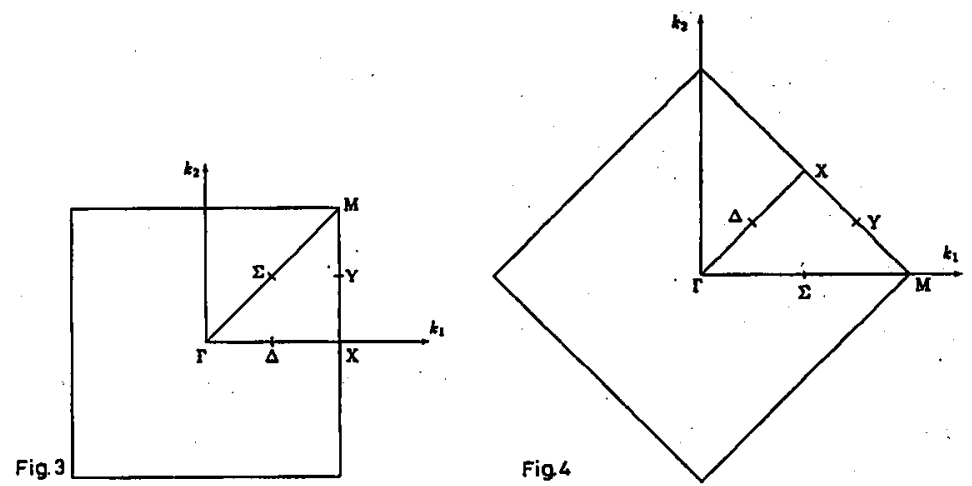

Fig. 3. Two-dimensional Brillouin zone for the orientations $\operatorname{sc}(001)$ and $\operatorname{bcc}(001)$; the coordinates of the high symmetry points are: $\Gamma(0,0), \Delta(\pi / 2 a, 0), X(\pi / a, 0)$, $Y(\pi / a, \pi / 2 a), M(\pi / a, \pi / a), \Sigma(\pi / 2 a, \pi / 2 a)$.

Fig. 4. Two-dimensional Brillouin zone for the orientations $\mathrm{fcc}(001)$; the coordinates of the high symmetry points are: $\Gamma(0,0), \Delta(\pi / 2 a, \pi / 2 a), X(\pi / a, \pi / a), Y(3 \pi / 2 a, \pi / 2 a)$, $M(2 \pi / a, 0), \Sigma(\pi / a, 0)$.

assembled in Table. The first Brillouin zones for the surface cuts considered are square in shape; they are shown in Figs. 3 and 4 with the points of high symmetry marked on them.

\section{The elements of the Hamiltonian matrix}

The diagonalization of the Hamiltonian (1) is equivalent to that of the following five-diagonal matrix of the $\operatorname{rank} L$ :

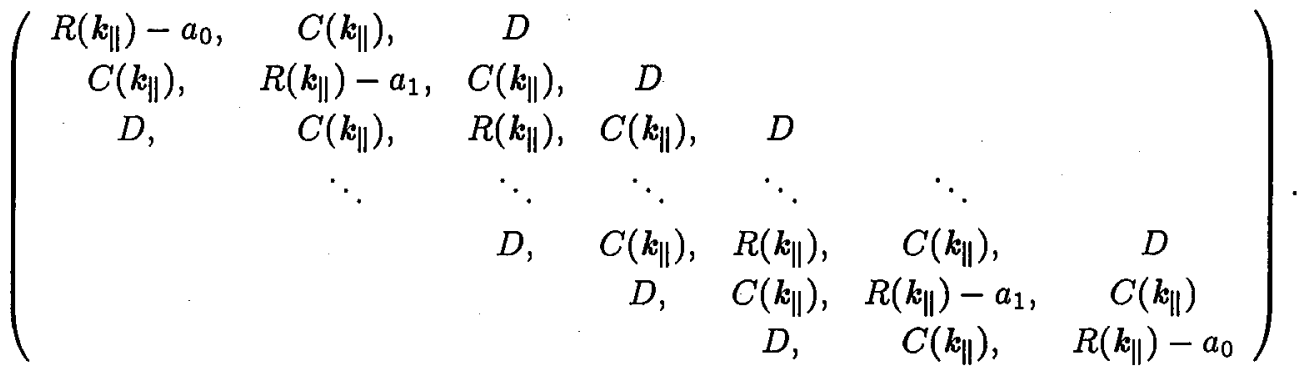

Its elements are defined as follows:

for the surface orientation $\mathrm{sc}(001)$

$$
\begin{aligned}
& R\left(k_{\|}\right)=2 S\left\{J_{1}\left[6-\Gamma_{0}\left(k_{\|}\right)\right]+J_{2}\left[12-\Gamma_{0}^{\mathrm{N}}\left(k_{\|}\right)\right]\right\}+g \mu_{\mathrm{B}}\left(\boldsymbol{H}^{\mathrm{eff}} \cdot \gamma\right), \\
& C\left(k_{\|}\right)=-2 S\left[J_{1}+J_{2} \Gamma_{1}^{\mathrm{N}}\left(k_{\|}\right)\right] \\
& D=0 \\
& a_{0}=2 S\left(J_{1}+4 J_{2}\right) \\
& a_{1}=0
\end{aligned}
$$


for the surface orientation $b c c(001)$

$$
\begin{aligned}
& R\left(k_{\|}\right)=2 S\left\{8 J_{1}+J_{2}\left[6-\Gamma_{0}^{\mathrm{N}}\left(k_{\|}\right)\right]\right\}+g \mu_{\mathrm{B}}\left(H^{\mathrm{eff}} \cdot \gamma\right), \\
& C\left(k_{\|}\right)=-2 S J_{1} \Gamma_{1}\left(k_{\|}\right), \\
& D=-2 S J_{2}, \\
& a_{0}=2 S\left(4 J_{1}+J_{2}\right), \\
& a_{1}=2 S J_{2}
\end{aligned}
$$

and for the surface orientation $\mathrm{fcc}(001)$

$$
\begin{aligned}
& R\left(k_{\|}\right)=2 S\left\{J_{1}\left[12-\Gamma_{0}\left(k_{\|}\right)\right]+J_{2}\left[6-\Gamma_{0}^{\mathrm{N}}\left(k_{\|}\right)\right]\right\}+g \mu_{\mathrm{B}}\left(H^{\mathrm{eff}} \cdot \gamma\right), \\
& C\left(k_{\|}\right)=-2 S J_{1} \Gamma_{1}\left(k_{\|}\right), \\
& D=-2 S J_{2} \\
& a_{0}=2 S\left(4 J_{1}+J_{2}\right), \\
& a_{1}=2 S J_{2} .
\end{aligned}
$$

Obviously, in the case of the orientation sc(001) the matrix (4) is in fact a tridiagonal matrix due to the circumstance that (see Fig. 2) the NNN neighbours in this case lie either in the $l$-th plane or the $(l \pm 1)$-th planes, whereas none lie in the $(l \pm 2)$-th planes. Whereas in the other two cases (see Fig. 2) NNN lie in the $l$-th and $(l \pm 2)$-th planes, and this last circumstance causes the matrix element $D$ to differ from zero. Moreover, in these two cases, only one NNN neighbour occurs in either of the ( $l \pm 2$ )-th planes "perpendicularly" above the $l$-th plane (its projection onto the surface plane coincides with the centre of the figure).

\section{Energy spectra extended over the Brillouin zone}

The energy dispersion relation one obtains by insertion of

$$
R\left(k_{\|}\right)-E\left(k_{\|}, k_{\perp}\right)=-2 C\left(k_{\|}\right) \cos \tau-2 D \cos 2 \tau
$$

into the matrix (4), where $\tau \equiv k_{\perp}$ is the wave vector component perpendicular to the surface of the film. With the formulae of the previous Section we get the following dispersion relations for the different surface orientations:

for the surface orientation $\mathrm{sc}(001)$

$$
\begin{aligned}
& E\left(k_{\|}, \tau\right)-g \mu_{\mathrm{B}}\left(\boldsymbol{H}^{\mathrm{eff}} \cdot \gamma\right)=4 S J_{1}\left(3-\cos k_{1}-\cos k_{2}-\cos \tau\right) \\
& \quad+8 S J_{2}\left[3-\cos k_{1} \cos k_{2}-\left(\cos k_{1}+\cos k_{2}\right) \cos \tau\right] ;
\end{aligned}
$$

for the surface orientation bcc(001)

$$
\begin{aligned}
& E\left(k_{\|}, \tau\right)-g \mu_{\mathrm{B}}\left(\boldsymbol{H}^{\mathrm{eff}} \cdot \gamma\right)=16 S J_{1}\left(1-\cos \frac{1}{2} k_{1} \cos \frac{1}{2} k_{2} \cos \tau\right) \\
& \quad+4 S J_{2}\left[3-\left(\cos k_{1}+\cos k_{2}\right)-\cos 2 \tau\right] ;
\end{aligned}
$$

and for the surface orientation $\mathrm{fcc}(001)$

$$
\begin{aligned}
& E\left(k_{\|}, \tau\right)-g \mu_{\mathrm{B}}\left(\boldsymbol{H}^{\mathrm{eff}} \cdot \boldsymbol{\gamma}\right)=4 S J_{1}\left[6-\left(\cos k_{1}+\cos k_{2}\right)\right. \\
& \left.\quad-4 \cos \frac{1}{2} k_{1} \cos \frac{1}{2} k_{2} \cos \tau\right]+4 S J_{2}\left(3-2 \cos k_{1} \cos k_{2}-\cos 2 \tau\right) .
\end{aligned}
$$

When determining the permitted branches of the spin wave energy spectra, since we have no knowledge of the quantized values of $k_{\perp}$, we have to rely on the 
diagonalization of the matrix (4) numerically by the use of an adequate procedure [13]. The relations (7) are helpful when it comes to determining the shape of the band boundaries (within which all the extended states have to be comprised). Our results are given in Figs. 5-7 consisting of energy spectra determined numerically for different values of the exchange integrals $J_{1}$ and $J_{\dot{2}}$. The reduced energy

$$
\frac{E\left(k_{\|}, \tau\right)-g \mu_{\mathrm{B}}\left(\boldsymbol{H}^{\mathrm{eff}} \cdot \boldsymbol{\gamma}\right)}{2 S J}
$$

is expressed either in units of $2 S J_{1}$ or in units of $2 S J_{2}$. We selected two extreme cases for which either $J_{1}$ or $J_{2}$ vanishes - thus eliminating either NN or NNN neighbourhoods from our considerations, and two intermediate cases differing by the sign of the coupling constant $J_{2}$ (ferromagnetic or antiferromagnetic, respectively, and retaining only ferromagnetic coupling $J_{1}$ ). Everywhere, the thin film is assumed to consist of five atomic planes $(L=5)$ - a particular value of $L$ chosen to provide good readability for the energy branches. The reduced energy spectra are determined for the individual surface cuts under consideration along the path connecting points of high symmetry of the Brillouin zone, as shown in Figs. 3 and 4 (the dense lines mark the boundaries of the band). Further on, we proceed to discuss the essentials of the spectra obtained versus theratio $J_{2} / J_{1}$.

The sc(001) spectrum (Fig. 5), in the case of interaction with NN neighbours only $\left(J_{2}=0\right)$, contains solely the energies of "bulk" states lying inside the band (one of the branches coincides with the lower boundary of the band and is unaccessible to discrimination). However, for $J_{2} / J_{1}= \pm 0.5$, two states lie beyond the band in certain regions of the Brillouin zone; further on, we shall demonstrate that these are surface states. Obviously, this proves that in the case of $\mathrm{sc}(001)$ orientation the existence of surface states is due to NNN interactions. Here, we achieve agreement with the work of Wallis et al. [4] (where the existence of surface states was proved for the segment $\Gamma X$ only). It is worth stressing that a potent argument in favour of the attribution of the surface bands to NNN interactions resides in the fact that a change in $J_{2}$ from positive (ferromagnetic) to negative (antiferromagnetic) causes the surface branches to be "ejected" from the region under the band into the region above the band. The physical significance of this becomes obvious once we note that the forming of a surface is equivalent to the rupture of NNN interactions with neighbours lying beyond the surface and the equilibrium energy of the spins at the surface becomes affected in a manner to render their reversal more easily or, respectively, more difficult.

We now proceed to an analysis of the spectra obtained for the surface orientation $\mathrm{fcc}(001)$ (Fig. 6). For the hypothetical situation when $J_{1}=0$ and $J_{2} \neq 0$ (Fig. 6b), we get a spectrum consisting of 5 branches contained within the band (the two lowest branches are not visible on the graph as they coalesce with the dense line marking the lower limit of the band); thus, all the states obtained are of the "bulk" type. For the inverse situation $\left(J_{2}=0, J_{1} \neq 0\right)$ (Fig. 6a), the spectrum exhibits two essentially novel aspects: firstly, its shape is no longer so regular as previously and in the $X M$ region the band, as it were, collapses and all the three "bulk" states become degenerate; secondly, the two lowest (surface) states descend 

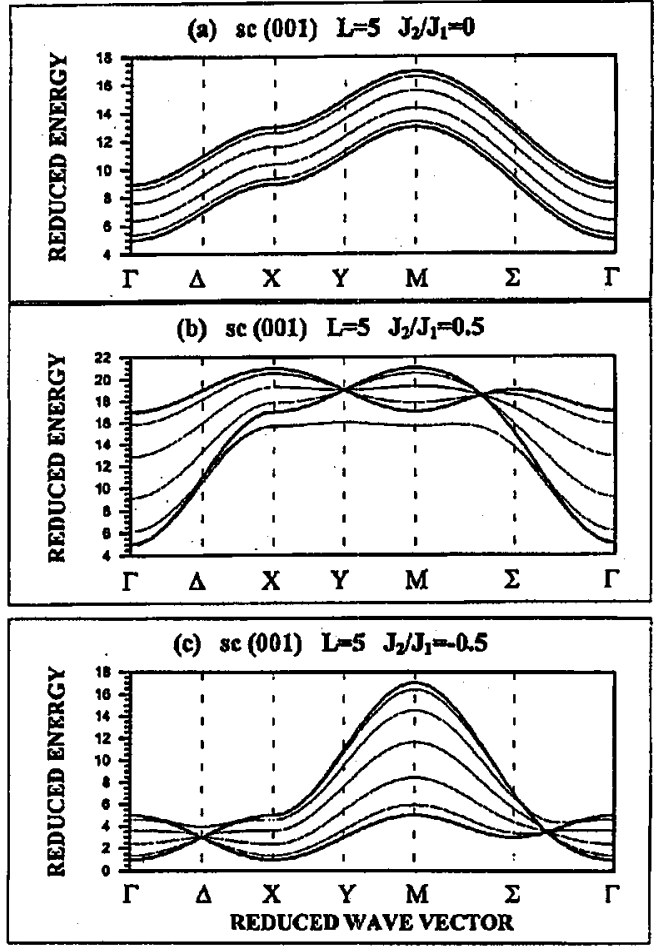

Fig. 5. The reduced spin-wave energy spectra (see Eqs. (7)) for a thin film (consisting of five layers, $L=5$ ) with surface orientation sc(001) versus the in-plane vector $k_{\|}$varying along the high symmetry path of Fig. 3, for indicated three values of the ratio $J_{2} / J_{1}$. The delicate lines are energy branches, whereas bold lines denote the boundaries of the band. One notes the existence of two surface branches. when the NNN exchange integral is non-zero $\left(J_{2} \neq 0\right)$; for the ferromagnetic case $\left(J_{2} / J_{1}=0.5\right)$ those two branches are disposed below the band, whereas for the antiferromagnetic case $\left(J_{2} / J_{1}=-0.5\right)$ they lie above the band.

below the band in the vicinity of the point $\Delta$ and stay there until we cross a point close to $\Sigma$. Moreover, although the inclusion of NNN interactions $\left(J_{2} \neq 0\right)$ does slightly modify the two surface branches, the modification to $J_{2}<0$ does not eject the two branches above the band as it was the case for the orientation $\mathrm{sc}(001)$. Thus, we are now justified in attributing the emergence of the two surface branches to $\mathrm{NN}$ interactions.

We may analyse the bcc(001) spectrum (Fig. 7) along similar lines. The spectra shown in Fig. 7 permit the conclusion that here, too, the presence of surface states is due to interaction with NN neighbours. For the case under consideration, we readily explain the "collapse" experienced by the spectrum in the region $X M$ (Fig. 7a): for the segment $X M$ the coordinate $k_{1}=\pi$ and (see Table) the structural factor $\Gamma_{1}\left(k_{\|}\right)$vanishes identically, leading to the vanishing of $C\left(k_{\|}\right)$in the matrix (4) (see Eq. 5b). And since the sub-case under consideration corresponds 

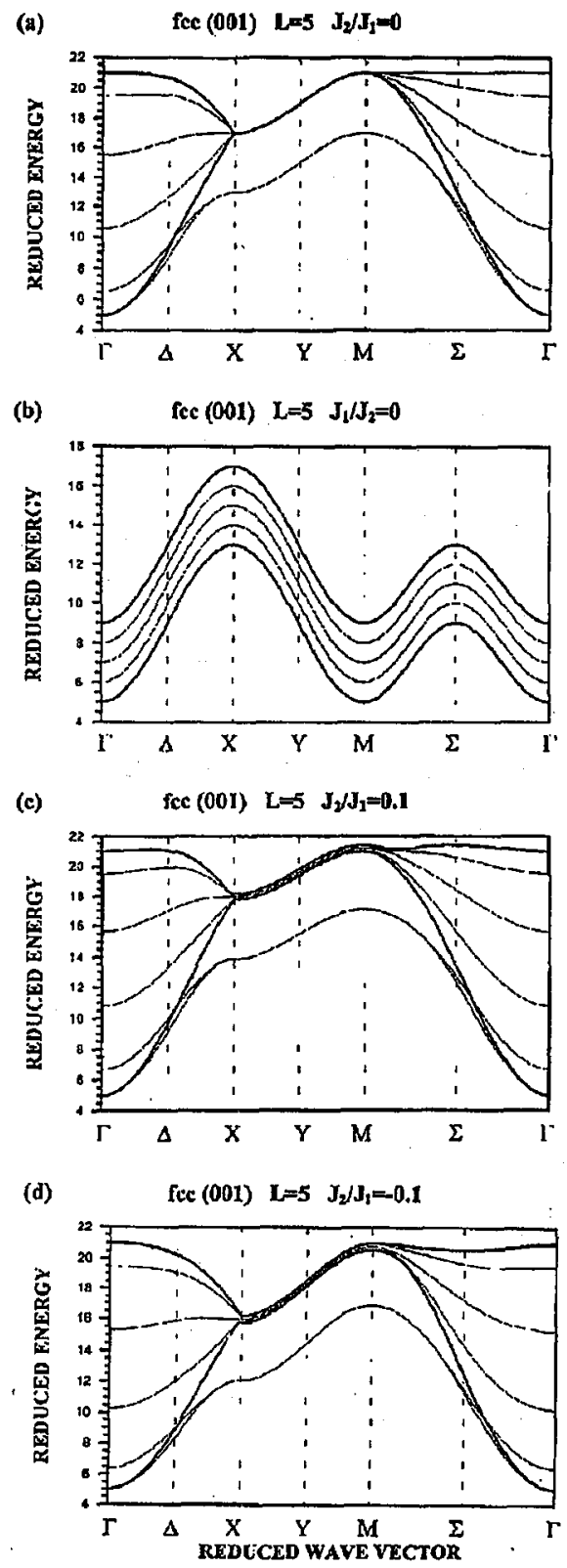

Fig. 6. The same as Fig. 5 for surface orientation $\mathrm{fcc}(001)$; the respective high symmetry path is shown in Fig. 4. Note that now the two surface branches exist only if $J_{1} \neq 0$ and disappear if $J_{1}$ is assumed (hypothetically) as equal to zero, and are practically insensitive to changes in $J_{2}$. 


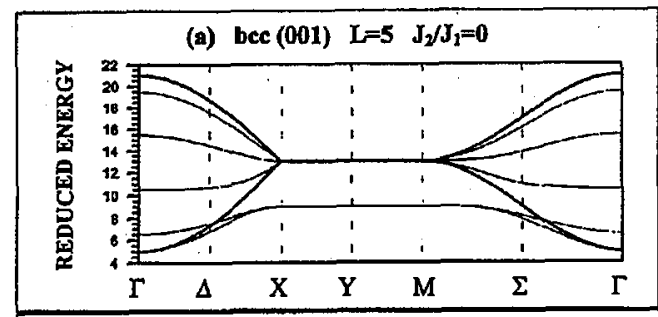

(b) $\operatorname{bec}(001) \quad L=5 \quad J_{2} / J_{1}=0.1$
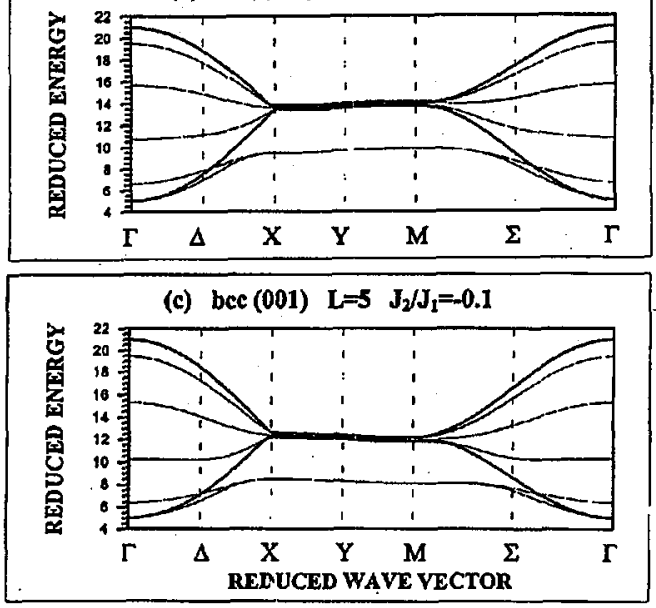

Fig. 7. The same as Fig. 6 for surface orientation bcc(001). Again, as in Fig. 6, two surface branches are very weakly sensitive to $J_{2}$.

to $J_{2} \equiv 0$, the matrix elements expressed by $D$ and $a_{1}$ also vanish. Finally, (4) becomes a diagonal matrix, consisting of elements of two kinds only: the "bulk" element $R\left(\boldsymbol{k}_{\|}\right)$is repeated $(L-2)$ times, and the boundary element $R\left(\boldsymbol{k}_{\|}\right)-a_{0}$ occurs twice. Accordingly, in Fig. 7a, we obtain in the region $X M$ but two energy levels: the one corresponding $(L-2)$-fold degenerate "bulk" excitation, and the other (lower lying) corresponding to twice degenerate "surface" excitation. Thus the effect of spectral "collapse" as observed at the edge $X M$ of the Brillouin zone is the result of "dynamical" vanishing of effective coupling between neighbouring planes of spins (as it occurs specifically for these propagation directions of the spin-wave in the plane of the film). The collapse in Fig. 6a (segment $X M$ ) and in Fig. 5 (particular points) is accessible to interpretation along similar lines.

Now, we shall illustrate the changes in profile of the two lowest lying spin wave states; for an example, we choose the orientation $f c c(001)$. We shall see that they in fact become of the surface type as their respective energy branches cross the lower boundary of the band (cf. Fig. 6). In Fig. 8 we show the profiles of the symmetric (energetically lowest) state $n=1$ and of the antisymmetric state $n=2$ in three points of the Brillouin zone: the point $\Gamma$ (the centre of the Brillouin zone), a point close to $\Delta$, and the point halving the segment $\Delta X$. The changes shown in Fig. 8 are highly instructive since they illustrate how the "bulk" mode (point $\Gamma$ ) 


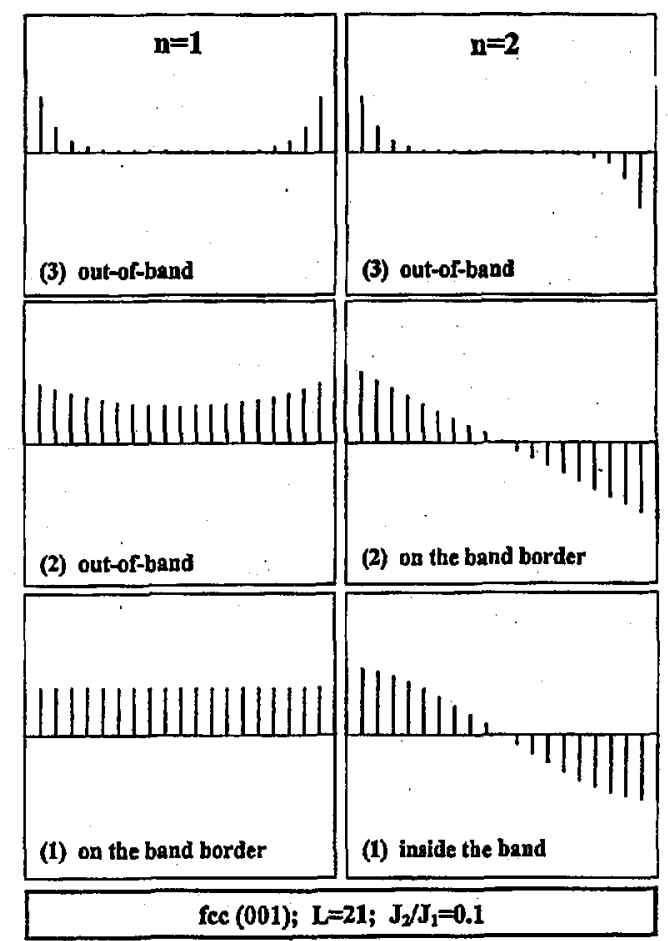

Fig. 8. The formation of surface localization of the two energetically lowest modes: symmetric $(n=1)$ and antisymmetric $(n=2)$, when their energy branches cross the lower bound of the bulk band (cf. Fig. 6c). The shapes of the modes are shown in their dependence on the wave-vector $k_{\| \mid}$along the high symmetry path shown in Fig. 4: (1) Brillouin zone centre $\Gamma,(2)$ vicinity of $\Delta$ and (3) halfway between $\Delta$ and $X$.

goes over into a surface mode on traversing an intermediate limiting bulk-surface stage. Similar evolutions occur for the other two surface orientations, but we shall not illustrate them here to save space.

\section{Conclusions}

When considering Sec. 4 we found that in the case of the orientation $\operatorname{sc}(001)$ the emergence of surface states was due to interaction with NNN neighbours, whereas in that of the orientations $b c c(001)$ and fcc(001) inversely, the existence of surface states was due to interaction with NN neighbours (interaction with NNN neighbours only modifies the energies of already extant surface states). To grasp the physical implications of the above, let us go back to Fig. 2 and analyse the distributions of the NN and NNN neighbours for the orientations under consideration. For the orientation sc(001) the NN neighbours lie in the $l$-th plane or are perpendicular thereto (one neighbour belonging to the plane $(l \pm 1)$ ). The NNN neighbours lie in the $l$-th plane or are "oblique" to it (four neighbours belonging to the plane $(l \pm 1))$. For fcc(001) the NN neighbours lie in the $l$-th plane and obliquely to it (four neighbours from the plane $(l \pm 1)$ ); the NNN neighbours lie 
in the $l$-th plane (four neighbours) and in the (l \pm 2$)$-th plane (one neighbour in the "perpendicular" configuration). For bcc(001), the NN neighbours lie obliquely (four neighbours from the plane $(l \pm 1)$ ); the NNN neighbours lie in the plane $l$ or perpendicularly to it (one neighbour from the plane $(l \pm 2)$ ).

Hence, we can draw the following conclusion, which holds in all the three cases considered: the emergence of surface branches in the spectra of thin films is due to the deficiency of ineractions with neighbours the position vectors of which are oblique to the film surface, irrespectively of whether these "oblique" interactions are of NN or NNN type. On a higher level of generality, with reference to arbitrarily remote neighbourhoods, the rule derived above can be formulated as follows: the act of formation of a surface in a solid consists in a rupture of the interactions between the surface atoms and the atoms removed; if the former comprise neighbours with position vectors oblique to the surface, their absence favours the emergence of surface states (for the well-defined directions of propagation along the surface) whereas the rupture of bonding with neighbours lying "perpendicularly" above the surface does not favour the emergence of surface states. Moreover, we can conclude that the influence of the ruptured "oblique" neighbours on the existence of surface states will be the greater the lesser is the inclination of their position vectors to the surface (since the departure from the neutral "perpendicular" configuration is now greater).

This rule appears to hold as well in cases involving other long-range interactions upward of NNN; for example, in magnetic materials, for the dipolar interactions. Applying the above rule to these interactions we gain a direct insight into the physical reasons for the existence of Damon-Eshbach modes (surface magnetostatic modes) [14]; we shall deal with the problem in a separate paper. (Our present interest in NNN interactions originates in our conviction that these long-range interactions may prove important as well in the studies of the properties of magnons in ferromagnetic multilayers [15]). Also we refrain here from a detailed analysis of some other results of interest obtained by us, thus the variability in shape of the energy band. A highly interesting aspect of these variations resides in the "collapse" of the band at the edges of the zone and the circumstance that this degenerating collapse does not bear on the surface states. These matters will be dealt with in a separate paper.

\section{References}

[1] W. Selke, in: Systems with Learning and Memory Abilities, Eds. J. Delacour, J.C.S. Lévy, North-Holland, Amsterdam 1988, p. 597.

[2] T. Tohyama, S. Maekawa, Phys. Rev. B 49, 3596 (1994).

[3] J.H. Han, D.J. Thouless, H. Hiramoto, M. Kohmoto, Phys. Rev. B 50, 11365 (1994).

[4] R.F. Wallis, A.A. Maradudin, I.P. Ipatova, A.A. Klochikhin, Solid State Commun. 5, 89 (1967).

[5] P.K. Schwob, M. Tachiki, E.G. Everett, Phys. Rev. B 10, 165 (1974).

[6] H. Puszkarski, Phys. Status Solidi 22, 355 (1967).

[7] J.C. Lévy, E. Ilisca, J.L. Motchane, Phys. Rev. B 5, 1099 (1972). 
[8] Diep-The-Hung, Phys. Status Solidi B 82, 383 (1977).

[9] J.R. Banavar, F. Keffer, Phys. Rev. B 17, 2974 (1978).

[10] I. Harada, O. Nagai, Phys. Rev. B 19, 3622 (1979).

[11] A. Duda, Phys. Status Solidi B 138, 509 (1986).

[12] H. Puszkarski, Prog. Surf. Sci. 9, 191 (1979).

[13] W.H. Press, B.P. Flannery, S.A. Teukolsky, W.T. Vetterling, Numerical Recipes in $C$, Cambridge University Press, Cambridge 1988.

[14] R.W. Damon, J.R. Eshbach, J. Phys. Chem. Solids 19, 308 (1961).

[15] H. Puszkarski, Surf. Sci. Rep. 20, 45 (1994). 